\title{
How are private medical colleges collaborating with the National TB Control Programme in Nepal, a qualitative study
}

\author{
Ingunn Harstad ${ }^{1,2 *}$, Chandra Mandil Yogal ${ }^{3}$ and Jennifer Infanti ${ }^{1}$ \\ ${ }^{1}$ Department of Public Health and Nursing, Faculty of Medicine and Health Sciences, Norwegian University of Science and Technology, NO 7489 Trondheim, Norway \\ ${ }^{2}$ Department of Pulmonary Medicine, St Olavs University Hospital, po box3250 Sluppen, N-7006 Trondheim, Norway \\ ${ }^{3}$ Department of Community Program, DHulikhel Hospital, Kathmandu University Hospital, Dhulikhel, Kavre, Nepal
}

\begin{abstract}
Background: Tuberculosis (TB) is an enduring public health problem in Nepal. This study aimed to uncover the experiences and attitudes of health workers at Private medical colleges (PMCs) towards the National TB Control Programme (NTP).

Methods: Strategic and convenience sampling were used to identify health personnel participating in TB care at PMCs affiliated to Kathmandu University. Three focus group interviews were held with 19 participants. Audio recordings of the discussions were transcribed, and thematic analysis was conducted.

Results: The PMCs treat many TB patients and regularly refer patients to DOTS clinics for treatment, but the PMCs don't feel well integrated into the NTP. There are gaps between the NTP programmes/ guidelines and clinical realities, and the PMCs would like to participate in developing national guidelines and the NTP`s monitoring and evaluation initiatives. There is also a lack of communication within and between levels. Clear lines of management and responsibility, and more training of staff at all levels, are wanted.
\end{abstract}

Conclusion: Increased involvement of PMCs in national TB control activities is important to improve TB care. Clearer guidelines from the government about the involvement of PMCs in TB control are also needed, as is better collaboration between the NTP and PMCs.

\begin{abstract}
Abbreviations: TB: Tuberculosis, NTP: The National Tuberculosis Control Programme, PMC: Private Medical College, DOTS: Direct observed treatment short course (how TB is treated). The same abbreviation is used about the DOTS strategy, a 5point strategy for TB management, WHO: World Health Organisation, UNICEF: United Nations Children's Fund, FGI: Focus group interview, KU: Kathmandu University, PTB: Pulmonary tuberculosis, ETB: Extrapulmonary tuberculosis, MDR-TB: Multi drug resistant tuberculosis, PCR: polymerase chain reaction, AFB: acid fast bacilla
\end{abstract}

\section{Background}

In Nepal, tuberculosis (TB) is a profound health issue. An estimated $45 \%$ of the population of 27 million is infected with TB, and the number of active TB cases is around 60000. The yearly incidence is approximately 45000, with 5000 deaths [1,2]. In 2013, 35000 persons were registered with TB disease. Globally, TB and HIV co-infections are common, with each disease facilitating the progress of the other. In Nepal, approximately $2 \%$ of TB patients are known to be HIV-positive. However, HIV status is only known for $11 \%$ of all TB patients [2].

Nepal has made significant progress in confronting the heavy disease burden of TB. As early as 1956, the government, with support from the World Health Organization (WHO) and the United Nations Children's Fund (UNICEF), initiated the Tuberculosis Control Programme to prevent new cases of tuberculosis. At this time, diagnostic and treatment services were provided by the Central Chest Clinic. Today the National Tuberculosis Center includes the Central Chest Clinic and the National
Tuberculosis Control Programme (NTP). In 1996, the international DOTS strategy, a 5-component strategy for TB management and control, was initiated in Nepal through the NTP. DOTS has proven to be effective with the number of deaths from TB declining from 9707 in 1990 to 5000 in 2013 [2]. The NTP provides guidelines about the diagnosis, registration, treatment and follow-up of TB cases [1]. It also clearly describes the organization and responsibilities for provision of $\mathrm{TB}$ care by health workers in the country [1]. Despite these efforts and the availability of effective treatment (DOTS), many patients diagnosed with TB in Nepal do not start treatment or do not complete treatment. In addition, the estimated burden of multidrug-resistant TB (MDRTB) in 2012 was $2,2 \%$ for new cases and $15 \%$ for retreatment cases [2].

Health service delivery in Nepal is provided through a mix of the private and public sectors. The NTP has primarily functioned through the public health sector but, later have extended its services through cooperation with communities and the private medical sector in order to provide free medical services. Many of Nepal's medical training colleges/teaching hospitals, are private institutions. Several studies

Correspondence to: Ingunn Harstad, Department of Public Health and Nursing Norwegian University of Science and Technology, MTFS, Norway, Tel: +47 99494996; E-mail: Ingunn.harstad@ntnu.no

Key words: TB control programme, medical college, public-private mix, Nepal

Received: November 04, 2017; Accepted: November 20, 2017; Published: November 22, 2017 
have been conducted in India to examine how private medical colleges (PMCs) there relate to the local National TB Control Programme [38]. In general, in these studies, the PMCs did not comply well with the NTP although improvements were seen following different systematic interventions. All of the studies in India were quantitative, and their designs did not facilitate explorations of the attitudes nor experiences of health care staff working in the area of TB control. No similar studies about the relationships between PMCs in Nepal and the NTP have been found in medical journal databases. As the private medical sector is an important player in health service provision in Nepal, a wellfunctioning partnership between this sector and the NTP is essential for achieving effective TB control in the country. However, the experiences and attitudes of health care workers from PMCs towards the NTP are unknown. Without such understanding, it is difficult to assess if the continuing high prevalence of TB in Nepal is related in any way to the non-compliance of PMCs with the NTP, or to any other aspect of the relationship between these entities. By extension, we do not know if the NTP should focus more on involving private medical institutions in its activities, how it could effectively engage such institutions, nor if this would result in better TB prevention, diagnosis, treatment, case notification and/or referral rates.

This study makes a preliminary contribution to filling the knowledge gaps noted above. It explores the current state of the relationship between PMCs and hospitals and the NTP, focusing on how the relationship is functioning, how it affects the quality of TB care in the country, and how it can be improved for the benefit of TB patients. This aim is pursued by exploring the experiences and attitudes of health care workers at PMCs towards the NTP.

\section{Methodology}

\section{Data collection and analysis}

A qualitative design was chosen for this study, specifically focus group interviews (FGIs), as we sought information about the experiences and attitudes of health care workers. Two facilitators guided the FGIs - the project leader, a Norwegian pulmonary specialist with clinical and research experience in tuberculosis both from Nepal and Norway, and a Nepali assistant with experience in public health and administration at a private hospital. The participants spoke mostly in English during the FGIs, although occasionally in Nepali. The FGIs were audiotaped and transcribed by the two facilitators; with the Nepali facilitator translating the Nepali statements to English. One FGI was held in April 2014, and two in August of the same year. Written notes were taken during the FGIs as well. An iterative process of thematic analysis was conducted by all authors over 8 months to develop and revise codes from the data sources, group the codes into themes, and generate explanations from the themes. A third researcher with extensive experience in qualitative research assisted in the analysis and writing processes. Combined, the researchers' diverse backgrounds and skills ensured a thorough interpretation of the findings.

\section{Study sites and participants}

Kathmandu University (KU), located in the city of Dhulikhel, Kavrepalanchok District, is a private institution with seven affiliated medical colleges throughout the country. Altogether 1100 medical students graduate through KU each year. Dhulikhel Hospital is the medical college hospital directly attached to KU. The Norwegian University of Science and Technology (NTNU) has a formal collaboration with KU/Dhulikhel Hospital, and the colleges included in this study were among those affiliated to $\mathrm{KU}$, building on this international partnership.
A mixture of strategic and convenience sampling techniques were employed to identify participants for the FGIs. First, invitations and study information were sent to all of KU's affiliated PMCs for FGIs to be held in April 2014. The invitations were directed to administrative persons responsible for $\mathrm{TB}$ education or $\mathrm{TB}$ service organization at the colleges; professors and clinicians responsible for TB education and/or clinical TB work; or other individuals related to TB work. The response from invitees was initially limited, thus a FGI was conducted with health care personnel from Dhulikhel Hospital. This FGI provided an opportunity to consult participants on how to revise the invitation to the other colleges and plan for the further discussion groups. A second invitation was thus circulated to the PMCs in August 2014 by the Dean of KU's School of Medicine Sciences. Following this, 14 individuals agreed to participate in two further FGIs.

The study therefore includes three FGIs with a total of 19 participants representing five different PMCs. The study participants included nurses (staff nurses and DOTS clinic nurses), microbiologists, both resident doctors and MDs in pediatric and internal medicine, and faculty members. We sought to mix different professions and staff from different PMCs in the groups. No administrative persons from the PMCs participated.

\section{Study instruments}

An interview guide was developed in advance of the FGIs and gradually improved over the course of the study. The following two questions elicited the main topics for discussion: (1) What are your experiences with the collaboration between you/your college and the NTP?; and (2) What are your suggestions for improving the collaboration? Additional probing questions were used to explore attitudes among staff, attitudes among heads of departments/leadership of the colleges and hospitals, and in regard to protocols, guidelines and the practicalities of following NTP guidelines in general.

\section{Ethics}

As no patients were involved in this study and no personal health information was requested, ethical clearance was not required from NTNU. Formal approval to conduct the study was given from the Institutional Review Board at Dhulikhel Hospital/Kathmandu University School of Medical Sciences. The National TB Center was also informed about the study. All participants signed an informed consent form and were told they could withdraw their consent or parts of their information at any time without consequence. After the interviews, the transcribed text was anonymized.

\section{Results}

Participants in the FGIs held diverse views and opinions about the NTP, ranging from general comments on how the DOTS programme is organized and working, to the more specific medical content of the programme and how the individual PMCs collaborate with the NTP. Despite challenges with the current collaboration, it was clear the PMCs want to be a part of the national programme; included in the guidelines for referral, treatment, surveillance and training; and involved in initiatives to further develop and plan the programme. In the remainder of this section the key findings from the FGIs are presented, focusing on the most commonly expressed opinions as well as contradictory and unexpected findings.

\section{The National TB Control Programme}

The NTP "is one of the most successful programmes in our country as a whole, and I think one of the most successful programmes within 
Southeast Asia. By following the NTP guidelines we have increased the [TB] cure rate" (FGI 1). This opinion, expressed by a doctor, was shared by the majority of participants in all of the FGIs. The overriding view was that the NTP has been a great success in Nepal, streamlining treatment regimens for $\mathrm{TB}$ patients and contributing to a greater cure rate. Free drugs for patients following the introduction of DOTS were also considered a great benefit of the programme.

Praise for the NTP, however, existed alongside confusion and discontent regarding the guidelines and protocols. Study participants mentioned that the programme is not always realistic when confronted with the practical or medical realities of life in Nepali communities. For example, the protocols suggest using some formulations of drugs for pediatric TB that are neither available in the DOTS clinics nor even in Nepal. Also, when patients experience side effects from drugs, it is difficult to acquire the standard alternative, single drug formulations, because the programme only suggests using combined drugs. Overall, the guidelines do not allow enough flexibility or give sufficient advice regarding difficult cases, comorbidities, drug reactions or side effects. For pediatric $\mathrm{TB}$ and spinal $\mathrm{TB}$, amongst other challenging cases, participants felt the DOTS-programme was not relevant at all given that these patients require longer treatment than the guidelines recommend. Guidelines for treatment of latent TB in children were also mentioned as lacking and desired.

Another frequently discussed problem with the diagnostic guidelines was their near exclusive focus on pulmonary TB (PTB) by sputum microscopy. As a consequence of this emphasis, one participant explained, "there are no proper guidelines for complicated cases of extrapulmonary TB (ETB)" (FGI 2). Another participant added his concern: "In my experience the NTP has not concentrated much [on] pediatric tuberculosis. Most of the time we have many difficulties diagnosing pediatric TB" (FGI 1). Again, with more complicated cases, the guidelines were felt to be lacking, only suggesting referral to a doctor or a hospital, and giving no further advice or information about what the doctor or the hospital should do to ensure effective treatment and care.

One study participant expressed that he would like to see "at least one sophisticated laboratory in each region that patients can reach within a short period and that can provide reports in a short time... and these laboratories should be set up in the private teaching hospitals" (FGI 1). In general, staff working at the PMCs wanted 'culture facilities' to diagnose sputum negative PTB or ETB, and perform susceptibility testing to diagnose MDR-TB. Thus far, only the National TB Centre has been analyzing cultures, and only for respiratory samples: "There are only a few laboratories with the facilities to culture mycobacteria" (FGI 1). Better diagnostic tools are also in great demand, as expressed clearly in the FGIs: "In [the] case of tuberculosis effusion we rely on the counts in the fluid and adenosid deaminase (ADA)...The polymerase chain reaction $(P C R)$ is a recent investigation and is not available everywhere (FGI 1). The new GenExpert machine, which can detect M.tuberculosis by genetic methods, holds potential for improving case detection and giving fast indications of Rifampicin resistant TB strains. However, to date, mainly governmental hospitals have been given these facilities. "Every time we send patients for sputum AFB (acid fast bacilla) we find negative results. So, we are thinking, if we have diagnosed patients symptomatically, let's send them for GenExpert first" (FGI 2). The PMCs would like the government to provide them with these machines too to improve their diagnostic processes.

\section{DOTS clinics}

A number of challenges were discussed in relation to DOTS clinics. One of the PMCs represented by our study participants does not have a DOTS clinic at the hospital and therefore all TB cases must be referred to the DOTS clinics closest to the patients' homes. To get free medicines to their in-patients this college must ask the various DOTS clinics. Such a process presents a variety of challenges; for example, "Once the patients are diagnosed, sometimes they [the DOTS clinic] ask for an AFB (acid fast bacilli) report, [they say this] should be included to get the medicine. But not all the patients can expectorate, they are not experiencing this problem. If there is no report of sputum the clinic hesitates to give medicine" (FGI 3). Both pediatric patients and patients with extrapulmonary TB often do not have a positive sputum test either, and some patients are too ill to be able to make a sputum test. Follow-up is also challenging with patients whom, for medical reasons, should be seen at the hospital but are required to go to the DOTS clinics to obtain the necessary drugs for treatment. This process is considered burdensome and a contributing factor for reduced treatment compliance.

The other PMCs represented in this study have DOTS clinics at their hospitals which are normally staffed by a nurse who counsels patients and then refers them to the clinics closest to their homes. Patients will typically receive drugs for 5-7 days at the hospital DOTS clinics, until they can reach their local DOTS clinics to continue with treatment. "We ask them [patients] to get follow-up through the health post but the problem is that most of them are not following [this advice]. So it would be better if those people who have been diagnosed in the hospital could be followed-up by the hospital, rather than going to the health post" (FGI 2). According to study participants, sometimes there is no staff present when a patient arrives at a health post, and the staff at these clinics requires more training in counselling and medical challenges, such as the side effects of drugs. Also, there are many DOTS clinics but patients can be too ill to walk or the clinic too far away: "If a patients' home is distant from a DOTS clinic then direct observed treatment is not possible" (FGI 3). This affects treatment completion rates. The study participants wanted more clinics or voluntary health workers to distribute drugs in patients' homes or in the community as part of the official DOTS programme. They also wanted PMCs to be able to treat and follow-up their patients at the hospitals instead of referring them.

\section{MDR-TB}

MDR-TB was one of the primary concerns of study participants, and was discussed at length, particularly in respect to diagnostic facilities, referral and follow-up procedures, and infection control.

None of the PMCs have special facilities to diagnose or treat MDR-TB patients, creating a variety of challenges to good care. Some PMC staff could not find suitably located referral centres for their MDR-TB patients; some had encountered doctors treating MDR-TB in their own ways (outside of the guidelines); and many mentioned that patients are started on treatment without a proper diagnosis. As mentioned previously, study participants wanted more referral centres in different parts of the country and to have GenExpert machines in their own hospitals to diagnose resistant strains, and also to be able to do cultures analyses. With such improvements, the PMCs felt they could treat patients with MDR-TB themselves or refer only a select few who needed specialized or individualized care.

Study participants also discussed common practices that increased the risk of developing MDR-TB like some doctors prescribing drugs 
for a few weeks or 1-2 months without ensuring adequate follow-up. In general, participants felt that follow-up on treatment regimens was not good enough and many DOTS clinics were located too far from where patients live. Also, over-the-counter sale of fluoroquinolones was believed to be creating resistant strains.

According to study participants, newly diagnosed patients might be put into the same room as MDR-TB patients and might therefore become infected by a resistant strain; also, hospital staff are sometimes infected by their patients. Equipment, like masks, and available isolation rooms or wards are greatly wanted and PMC staff felt such extra equipment and facilities should be provided by the NTCP: "Doctors must wear masks" (FGI 2). One participant felt that having a $\mathrm{TB}$ isolation room should be a prerequisite requirement for receiving affiliation by the NTCP and KU as a private medical college.

\section{Collaboration between PMCs and NTP}

Study participants expressed a variety of concerns about the management of PMCs and how this impacted TB care, as well as the nature of the relationship between PMCs and the NTP. A commonly held opinion was that the PMCs are managed by staff who do not have medical training and, as a result, an inadequate understanding of the clinical expression and management of medical problems, or how serious the TB epidemic is in Nepal. "Because the PMCs are run by nonmedical [staff], they do not know what MDR-TB is, what tuberculosis is or what is difficult in diagnosis. They cannot understand the gravity of the situation. And we cannot tell them, we cannot make them understand because it is very difficult" (FGI 3). Participants also felt that the management authorities of PMCs are not willing to send staff for training to maintain or update their skills unless they are instructed to do so by higher authorities, such as KU or governmental officials.

A similar concern mentioned by participants was the apparent information gap between those responsible for creating the national TB programme and the health care workers actually using national guidelines and tools. One informant explained that he had an old version of the control programme and had been teaching from that protocol until he realized his information was outdated during a visit from the NTP. He had a strong message for the NTP: "Once they have any [new] program, don't keep it separately, spread it out, everybody should know" (FGI 2). Similarly, participants felt the clinical guidelines were not well suited for them but were mainly made by people living and working in the capital city or even outside Nepal. They expressed a need for research and protocols adjusted to be relevant to the various contexts of Nepal. Also, before new protocols are made, participants felt there should be debates about the shortcomings of the current guidelines and implementation of the DOTS programme and how to improve both. The PMC staff would like to be involved when such new programmes are made.

Overall, participants felt there was a lack of communication between the NTP and the PMCs. Previously, the NTP related only to governmental hospitals, and the study participants still felt that the main focus of the NTP was on governmental hospitals or the DOTS clinics attached to some PMCs. According to participants, PMCs deal with TB largely on their own as they don't feel they are part of the national TB programme. They are also required to charge their patients a fee for sputum tests, which are free inside the NTP. Many of the PMC employees do not even know about the NTP, a reality that some participants considered one of "the main problems" (FGI 3) for improving TB care in Nepal.
Participants desired a relationship that involved them in more than free DOTS treatment - they felt they should also report cases to the NTP; there should be regular surveillance of their TB activities; and they would like the NTP to conduct external quality controls of their care and services. "I think they [the NTP] should change their attitudes towards private medical colleges. PMCs should be treated as the governmental hospitals" (FGI 3). Some study participants mentioned they would like the PMCs to be referral centres from primary health care, or regional referral centres. Others mentioned they would like more training at all levels of staff from doctors to voluntary health workers to laboratory staff. They felt the NTP should involve staff at PMCs in national workshops, courses and research.

\section{Discussion}

The aim of this study was to explore the experiences and attitudes of health care workers at PMCs towards the NTP, with a focus on how this collaboration could be improved. This was achieved by inviting health personnel from PMCs to focus group interviews. Based on our literature survey and discussions with involved people in Nepal we expected to find greater differences between practices at the various PMCs than were indicated in the FGIs. We anticipated that some PMCs would be working independently of the NTP and treating their own patients instead of referring them to the DOTS clinics as all of them were doing. We had also expected to see the PMCs using their own advanced guidelines or protocols for diagnosing and treating difficult cases, and anticipated stronger opinions about the division of responsibilities for TB care between the government and the PMCs. What we found is that PMCs treat many of Nepal's TB patients and regularly refer patients to DOTS clinics for treatment but do not feel well integrated into the NTP.

One of the focus areas of the Stop TB Strategy, the World Health Organization's strategy to dramatically reduce the global burden of TB by 2015 , is to contribute to national health system strengthening through involving all care providers $[9,10]$. In Nepal, the PMCs are already formally included in the national TB control strategy by having DOTS clinics at most of their hospitals or referring all patients to a nearby DOTS clinic. However, the medical staff at these institutions feels otherwise excluded from the national programme; the DOTS clinics are their only links to the NTP. There was a general perception amongst our study participants that it was unfair for the governmental hospitals only to benefit from the NTP with diagnostic facilities, equipment and training. PMC staff would also like to have access to these benefits and tools, especially diagnostic facilities for analyzing cultures and GenExpert technology. GenExpert has been introduced in eastern Nepal and has increased the number of laboratory verified cases of TB and suspected MDR-cases in that area [11]. The fact that GenExpert is provided mainly to the governmental hospitals to date, and that culture and susceptibility testing is available only at the National TB Centre, was mentioned frequently by participants in this study as evidence that the PMCs are not treated on an equal basis as government hospitals.

Previous studies on the relationship between private medical colleges and NTPs are sparse, in Nepal and elsewhere. Most related studies from other countries are on partnerships between private practitioners $[8,12]$ or between private and public hospitals [5]. One study from Nepal on a private-public partnership in TB control concluded that a strong NTP, confidence between the partners, and a belief that the partnership could succeed were all necessary ingredients for a constructive relationship [13]. An interface between the partners 
in such collaborations is also necessary to overcome potential suspicion between the partners [13]. The same study discussed the necessity to build trust and nurture relationships. In a study on a private-public mix of TB care in Indonesia, many of the private practitioners treated their own patients without referring them [12]. While this is not the case in Nepal, another problem mentioned both in our study and in Indonesia is that DOTS clinics only give free medicines to patients with positive sputum tests. For pediatric patients, ETB and sputum negative pulmonary $\mathrm{TB}$, health care providers have to struggle to obtain free drugs. In addition, both diagnostic and treatment guidelines are mainly made for sputum positive pulmonary TB and are not suited for the more complicated cases. Thus, there are gaps between the clinical programmes/guidelines and available training, and the realities that staff encounter.

Staff at the PMCs would like to participate in developing guidelines that better meet their needs, but face a number of obstacles in this regard. Where the NTP has invited PMC staff to such workshops in the past, attendance has been low, likely due to staff perception that their managers will not permit them to leave regular work duties for training or workshops. The same problem was mentioned in a previous study in Nepal on linking private and public sectors in tuberculosis treatment [14]. However, in this study, when a workshop on TB control was initiated by the national medical association, many doctors arrived. This study also speculated that private doctors attended on their own initiative when they did not rely on permission from the hospital administration. The participants in our study perceived that because the leadership of the PMCs have limited medical knowledge they are not interested in TB control and not willing to send staff for workshops. Other obstacles for PMC staff include not being invited to courses or not receiving information or invitations due to poor communication at the hospital level.

\section{Consequences of the lack of collaboration}

Overall, the results of our FGIs suggest the NTP and PMCs have to work harder to create the necessary trust and understanding for a good collaboration. The current lack of collaboration between the NTP and the PMCs has many potential consequences, such as underand over-diagnosis of TB cases; increased rates of treatment default; undiagnosed cases of MDR-TB; and increasing numbers of MDRTB. Patients' rights for tuberculosis care, as described in the patients charter for tuberculosis care [15], are violated on the right to care, dignity, information and security by the lack of collaboration between the PMCs and NTP. This is also a poor model for medical students.

\section{Responsibilities and permissions}

The first point of the Stop TB Strategy is to enhance the DOTS strategy, and this requires strong political commitment at the national level [16]. In Nepal, the government gives permission to the private medical colleges through the Department of Industries. However, the university hospitals are registered by the Ministry of Education and the Ministry of Health and Population. Through Kathmandu University, the PMCs are given permissions to run their medical schools. According to the participants in our FGIs the governing bodies of the PMCs are not interested in TB care, and require incentives to implement better $\mathrm{TB}$ care in their hospitals. The government could set standards of TB care that must be fulfilled by the PMC hospitals in order to be allowed to run, such as collaborating with the NTP, sending staff for training, and following national guidelines. Training for all kinds of health care personnel is an essential part of Stop TB Strategy, both under the DOTS expansion initiative and to ensure health system strengthening [16]. The requirement to follow these standards could be evaluated before the renewal of licenses. To obtain licenses for their medical schools, there should also be infection control measures so students are not infected, and standards for TB control training. Finally, some financial and technical support for TB care should be provided to PMCs from the government.

\section{Strengths and limitations of the study}

The diversity of health care personnel who participated in the FGIs ensured a broad representation of perspectives in this study. Participants represented five different PMCs, both from the capital city and rural areas, and a number of different professions. They were eager to talk. However, we were not able to obtain the views of hospital owners as none were present, thus we cannot say what their opinions are except from the perspectives of their staff. The value of not having hospital leaders present in the group discussions, however, was that staff was able to speak more freely. Faculty members were present, but no senior professors participated in the FGIs; it would be valuable to obtain the opinions of professors in a future study as they are influential players in the PMCs. This study focused on the experiences of staff at PMCs, thus we do not know what kinds of responses the NTP would have given on the topics discussed. This is an area for further research.

\section{Conclusion}

Private medical colleges in Nepal are not properly included in the NTP but still treat many TB patients. This may lead to improper diagnosis, treatment failure, and increases in MDR-TB cases. Improved communication, involvement of the PMCs in the planning of national protocols, inclusion of PMCs in national training programmes, and using PMCs as referral hospitals, could all improve TB care and control in Nepal. Such efforts require governmental involvement in giving directives (legislation), permissions and resources, and interest and willingness from both the NTP and PMCs. This study has generated a number of directions for future research. In particular, staff working at the National TB Center should be consulted about improving their collaboration with PMCs and patients' perspectives on how services could best be organized should be sought.

\section{Authorship and contributions}

This project was started after discussions with NTP Nepal. IH had overall responsibility for the project. However, Dr. Rajendra Koju at KU, Aslak Steinsbekk at NTNU, CY and JJI participated in project planning. CY also participated in the data collection, analysis and writing, and JJI supervised the analysis and writing processes.

\section{Acknowledgements}

We would like to thank Dr Rajendra Koju and Aslak Steinsbekk for important input in the project planning processes and Roshan Kuman Mahato for reading through the manuscript and giving important input. We also thank Samita Giri who took notes during the FGIs. Without the participants in the FDIs the study could not have been done. We are very grateful to them all. There was no external funding for this study.

\section{Competing interests}

The authors declare that there is no competing interests regarding the publication of this paper.

\section{Ethics approval}

As no patients were involved in this study and no personal health information was requested, ethical clearance was not required 
from NTNU. Formal approval to conduct the study was given from the Institutional Review Board at Dhulikhel Hospital/Kathmandu University School of Medical Sciences. The National TB Center was also informed about the study.

\section{Consent to participate}

All participants signed an informed consent form and were told they could withdraw their consent or parts of their information at any time without consequence.

\section{References}

1. NTC (2015) National Tuberculosis Programme Nepal. Annual Report 2012/2013. National Tuberculosis Center, Bhaktapur, Nepal.

2. WHO (2015) WHO Tuberculosis Country profile-Nepal.

3. Agarwal SP, Tonsing, J, Chauhan LS (2005) Tuberculosis control in India. Directorate General of Health Services, Ministry of Health and Family Welfare, New Delhi.

4. Quazi TA, Sarkar S, Borgohain G, Sreenivas A, Harries AD, et al. (2012) Are all patients diagnosed with tuberculosis in Indian medical colleges referred to the RNTCP? Int J Tuberc Lung Dis. 16: 1083-1085.

5. Arora V, Jaiswal AK, Gupta S, Gupta MB, Jain V, et al. (2012) Implementation of RNTCP in a private medical college: five years' experience. Indian J Tuberc 59: 145150. [Crossref]

6. Tonsing J, Mandal PP (2003) Medical colleges' involvement in the RNTCP: current status. J Indian Med Assoc 101: 164-166. [Crossref]
7. Ambe G LK, Dholakia Y (2005) Every provider counts: effect of a comprehensive public-private mix approach for TB control in a large metropolitan area in India. Int $J$ Tuberc Lung Dis 9: 563-568.

8. Lal SS, Sahu S, Wares F, Lönnroth K, Chauhan LS, et al. (2011) Intensified scale-up of public-private mix: a systems approach to tuberculosis care and control in India. Int $J$ Tuberc Lung Dis 15: 97-104. [Crossref]

9. WHO (2008) Contributing to health system strengthening. Guiding principles for national tuberculosis programmes.

10. WHO (2014) Public-Private Mix (PPM) for TB Care and Control. Tuberculosis (TB).

11. Creswell J, Rai B, Wali R, Sudrungrot S, Adhikari LM, et al. (2015) Introducing new tuberculosis diagnostics: the impact of Xpert( $(\mathbb{B})$ MTB/RIF testing on case notifications in Nepal. Int J Tuberc Lung Dis 19: 545-551. [Crossref]

12. Watkins RE, Feeney KT, Bakar OA, Plant AJ (2006) Joining the DOTS in Bali: private practitioners' perceptions of tuberculosis control. Int J Tuberc Lung Dis 10:988-994.

13. Newell JN, Pande SB, Baral SC, Bam DS, Malla P (2005) Leadership, management and technical lessons learnt from a successful public-private partnership for TB control in Nepal. Int J Tuberc Lung Dis 9: 1013-1017.

14. Hurtig AK, Pande SB, Baral SC, Newell J, Porter JD, et al. (2002) Linking private and public sectors in tuberculosis treatment in Kathmandu Valley, Nepal. Health Policy Plan 17: 78-89.

15. WHO (2006) The Patients charter for Tuberculosis Care. Tuberculosis care.

16. WHO (2015) The End TB Strategy. Tuberculosis care.

Copyright: ( 2017 Harstad I. This is an open-access article distributed under the terms of the Creative Commons Attribution License, which permits unrestricted use, distribution, and reproduction in any medium, provided the original author and source are credited. 